\title{
Nasal Administration of Stem Cells: A Promising Novel Route to Treat Neonatal Ischemic Brain Damage
}

\author{
CINDY T.J. vAN VELTHOVEN, ANNEMIEKE KAVELAARS, FRANK vAN BEL, AND COBI J. HEIJNEN
}

Laboratory for Neuroimmunology and Developmental Origins of Disease [C.T.J.V., A.K., C.J.H.], Department of Neonatology [C.T.J.V., F.B.], University Medical Center Utrecht, 3584 EA Utrecht, The Netherlands

\begin{abstract}
Mesenchymal stem cell (MSC) transplantation is a promising therapy to regenerate the brain after an ischemic event. We investigated the possibility to use the nasal route as a noninvasive method to repair the neonatal damaged brain. Nine-day-old mice underwent cerebral hypoxia-ischemia (HI), and MSCs were transplanted intranasally $10 \mathrm{~d}$ after HI. At $28 \mathrm{~d}$ after HI, MSCs were still present in the affected hemisphere but had not differentiated into cerebral cell types. Intranasal MSC treatment significantly improved sensorimotor function in the cylinder rearing test at 21 and $28 \mathrm{~d}$ after HI. Furthermore, intranasal MSC treatment decreased gray and white matter area loss when determined $28 \mathrm{~d}$ after $\mathrm{HI}$ by 34 and $37 \%$, respectively. MSC cultured in vitro with brain extracts obtained $10 \mathrm{~d}$ after HI, responded to the ischemic brain by up-regulation of several growth factors, including fibroblast growth factor 2 and nerve growth factor in comparison with brain extracts of sham-operated controls. In conclusion, MSC can reliably be delivered to the brain via the nasal route to induce functional recovery and a reduction in brain lesion size. We propose that MSC function by stimulating endogenous cerebral repair by adapting their secretion profile to the ischemic brain leading to up-regulation of repair promoting factors. (Pediatr Res 68: 419-422, 2010)
\end{abstract}

$\mathrm{N}$ eonatal hypoxia-ischemia (HI) causes severe brain damage and remains a major cause of neonatal morbidity and mortality (1). At present, treatment options for neonatal HI brain damage are very limited (2). Recent clinical trials have shown that hypothermia (cooling of the brain by $2-4^{\circ} \mathrm{C}$ ) has modest effects on outcome $(3,4)$.

The therapeutic potential of mesenchymal stem cells (MSCs) has been studied in several rodent models of ischemic brain injury (5-7). The data show that MSC transplantation improves functional outcome and can also restore brain structure (7-9). Recently, we and others have shown that transplantation of bone marrow-derived MSC markedly improves functional outcome after neonatal HI in rodents (7,9-11). Furthermore, we have shown that intracranial MSC transplantation after neonatal HI enhanced neurogenesis and oligodendrogenesis and reduced lesion volume (7). It has been suggested that MSC promotes repair of injured tissue by replacement of damaged cells. However, MSC also secrete growth and differentiation factors that could stimulate endogenous repair processes $(12-14)$.

Received April 23, 2010; accepted June 30, 2010.

Correspondence: Cobi J. Heijnen, Ph.D., University Medical Center Utrecht, Room KC03.068.0, Lundlaan 6, 3584 EA Utrecht, The Netherlands; e-mail: C.Heijnen@umcutrecht.nl

Supported by the Wilhelmina Children's Hospital Research fund.
The intracranial route of administration is frequently used in rodent studies, but for clinical application, a less invasive route is preferred. Although i.v. MSC delivery can be effective, the loss of cells in peripheral organs before they reach the brain is an important possible drawback. Existing evidence indicates that after intranasal delivery, MSCs are capable of crossing the cribiform plate and migrate throughout the brain using the rostral migratory stream (15). The goal of this study was to determine whether intranasal delivery of MSC can be used as the route of entry to treat neonatal hypoxic-ischemic brain damage.

\section{MATERIALS AND METHODS}

MSC. Bone marrow from femur and tibia of 6- to 8-wk-old C57B1/6-Tg (UBC-GFP) 30Scha/J mice (Jackson Laboratories, Bar Harbor, ME) was cultured in DMEM $/ 15 \%$ fetal bovine serum as described (7). Cells were negative for myeloid and hematopoietic cell lineage specific antigens and positive for Sca-1, CD90, CD29, CD44, and MHC class I (7). Before intranasal application, cells were labeled with $\mathrm{PKH}-26$ according to manufacturers' instructions (Sigma-Aldrich Chemical Co., Steinheim, Germany).

Animals. The animal committee of Academic Biomedical Center Utrecht approved all experiments. At postnatal d 9 (p9), C57B1/6 mice pups of both sexes underwent $\mathrm{HI}$ by permanent right common carotid artery occlusion under isoflurane anesthesia [3\% induction and $1 \%$ maintenance in $\mathrm{O}_{2}: \mathrm{N}_{2} \mathrm{O}(1: 1)$ ] followed by exposure of $10 \%$ oxygen in nitrogen for $45 \mathrm{~min}(16)$. This procedure induced a lesion involving hippocampus, neocortex, and striatum. Pups from at least three different litters were used in each experimental group, both genders were equally distributed among experimental groups, and data were obtained in at least two independent experiments. All analyses were performed in a blinded set up. Mortality $(\sim 10 \%)$ only occurred immediately after HI.

At $10 \mathrm{~d}$ after $\mathrm{HI}$, MSC or vehicle was delivered intranasally in awake animals. Thirty minutes before MSC or vehicle administration, two doses of $3 \mu \mathrm{L}$ hyaluronidase (total $100 \mathrm{U}$; Sigma-Aldrich Chemical Co.) in PBS were applied to each nostril and spontaneously inhaled (15). Subsequently, a total of $5 \times 10^{5} \mathrm{MSC}$ in $12 \mu \mathrm{L}$ PBS or vehicle were administered as two doses of $3 \mu \mathrm{L}$ applied to each nostril. Animals were killed at $28 \mathrm{~d}$ after $\mathrm{HI}$ and perfused with $4 \%$ paraformaldehyde in PBS. Brains were embedded in paraffin or cryoprotected in $20 \%$ sucrose and embedded in Tissue-Tek O.C.T. compound (Sakura Finetek, Zoeterwoude, The Netherlands).

Functional outcome. The cylinder rearing test was used to assess forelimb use asymmetry at 10,21 , and $28 \mathrm{~d}$ after HI. The weight-bearing forepaw(s) to contact the wall during a full rear was recorded as left (impaired), right (nonimpaired), or both. Paw preference was calculated as [(nonimpaired impaired)/(nonimpaired + impaired + both) $] \times 100 \%(7,17)$.

Histology. Coronal paraffin sections $(8 \mu \mathrm{m})$ were incubated with mouse anti-microtubule-associated protein (MAP2; Sigma-Aldrich Chemical Co.) or mouse anti-myelin basic protein (MBP; Sternberger Monoclonals, Lutherville, $\mathrm{MD}$ ), and binding was visualized with a Vectastain $\mathrm{ABC}$ kit (Vector

Abbreviations: BDNF, brain-derived neurotrophic factor; FGF, fibroblast growth factor; HI, hypoxia-ischemia; MAP2, microtubule-associated protein-2; MBP, myelin basic protein; MSC, mesenchymal stem cell; NGF, nerve growth factor; NT3, neurotrophin-3; SDF, stromal cell-derived factor 
Table 1. Primers used for quantitative real-time RT-PCR

\begin{tabular}{llll}
\hline Symbol & RefSeq & \multicolumn{1}{c}{ Forward $\left(5^{\prime} \rightarrow 3^{\prime}\right)$} & \multicolumn{1}{c}{ Reverse $\left(5^{\prime} \rightarrow 3^{\prime}\right)$} \\
\hline$\beta$-actin & NM_007393 & AGAGGGAAATCGTGCGTGAC & CAATAGTGATGACCTGGCCGT \\
BDNF & NM_007540 & CACATTACCTTCCAGCATCTGTTG & ACCATAGTAAGGAAAAGGATGGTCAT \\
CXCR4 & NM_009911 & CATAATCTCTTCATTCCGAGGAG & CTACCACCATTTCAGGCTTTG \\
FGF2 & NM_008006 & GCGAGAAGAGCGACCCACAC & GAAGCCAGCAGCCGTCCATC \\
GAPDH & NM_008084 & TGAAGCAGGCATCTGAGGG & CGAAGGTGGAAGAGTGGGAG \\
IL1 $\beta$ & NM_008361 & TCTATACCTGTCCTGTGTAATG & GCTTGTGCTCTGCTTGTG \\
IL6 & NM_031168 & CCGCTATGAAGTTCCTCTCTGC & AGTGGTATCCTCTGTGAAGTCTCC \\
NGF & NM_013609 & ACGGGCAGCATGGTGGAG & TGTAGAACAACATGGACATTACGC \\
NT3 & NM_008742 & CCCTGGAAATAGTCACACGGATG & ATGCTGTTGCCTTGGATGCC \\
SDF1 & NM_021704 & CGGTGTCCTCTTGCTGTCC & CTCTGGCGATGTGGCTCTC \\
TGF $\beta 1$ & NM_011577 & GTGACAGCAAAGATAACAAAC & CTGAAGCAATAGTTGGTATCC \\
\hline
\end{tabular}

GAPDH, glyceraldehyde 3-phosphate dehydrogenase.

Laboratories, Burlingame). Brain damage was analyzed at a location equivalent to $-1.58 \mathrm{~mm}$ from bregma in adult mice by outlining both hemispheres on full section images using ImageJ software (National Institutes of Health, Bethesda, MD; http://rsb.info.nih.gov/ij/, 1997-2007). Ipsilateral MAP2 area loss was calculated as follows: [1 - (area ipsilateral MAP2 staining/area contralateral MAP2 staining)] times $100 \%$. Similarly, MBP area loss was determined (16).

To determine whether transplanted MSC differentiated into cerebral cell types, frozen sections $(10 \mu \mathrm{m})$ were incubated with mouse anti-NeuN, mouse anti-S100 $\beta$ (Chemicon, Temecula, CA) or rabbit anti-Iba1 (Wako, Richmond, VA) followed by AlexaFluor-488-anti-IgG (Molecular Probes, Eugene, OR).

MSC gene expression profile after culture with ischemic brain extracts. To assess whether MSC can sense and respond to signals provided by the ischemic brain, MSC gene expression was performed after culturing MSC in vitro with extracts from the brain from sham animals and animals after HI (13). Brains were collected from HI or sham-operated animals at $10 \mathrm{~d}$ after surgery. A standardized section of the ischemic hemisphere, centered at the HI lesion (bregma $-2 \sim 0 \mathrm{~mm}$ ), was obtained by dissection on ice. Brain sections were homogenized at $150 \mathrm{mg} / \mathrm{mL}$ in knockout DMEM (Invitrogen) and clarified by centrifugation for $10 \mathrm{~min}$ at $10.000 \times \mathrm{g}$ at $4^{\circ} \mathrm{C}$. Protein concentration was determined using a protein assay (BioRad, Hercules, CA) with BSA as standard and stored at $-80^{\circ} \mathrm{C}$ until use. Approximately, $1 \times 10^{6}$ MSC per well were seeded in six-well plates in complete MSC medium. After $24 \mathrm{~h}$, culture medium was replaced with knockout DMEM containing brain extract (final concentration $1 \mathrm{mg}$ protein/mL) from $\mathrm{HI}+$ vehicle-treated or $\mathrm{HI}+$ MSC-treated ischemic brain extract ( $n=4$ per condition). After $72 \mathrm{~h}$ in culture, total RNA was isolated from the MSC using the RNeasy mini kit (Qiagen, Venlo, The Netherlands) and transcribed to cDNA using oligo (dT), dNTP mix, first-strand buffer, DTT, RnaseOUT, and Superscript III (Invitrogen). Gene expression changes in several growth factors (Table 1) were measured using quantitative RT-PCR with SYBR green mastermix on the BioRad iQ5. Data were normalized using multiple housekeeping genes and analyzed by comparing $2^{-[\text {Delta]Ct }}$ of the normalized data. Fold changes were calculated relative to MSC cultured in brain extracts from sham-operated animals and killed at $10 \mathrm{~d}$ after HI.

Statistical analysis. Data are presented as mean \pm SEM and analyzed by two-way analysis of variance with Bonferroni posttests, and $p \leq 0.05$ was considered significant.

\section{RESULTS}

MSC in the ischemic brain after nasal application. Mice underwent $\mathrm{HI}$ on p9, and PKH 26-labeled MSC were administered intranasally at d 10 after HI. At d 28 after HI, which is $18 \mathrm{~d}$ after intranasal administration, MSC could still be detected in the brain (Fig. 1). Nasally applied MSC were present in several layers of the olfactory bulb but predominantly in the glomerular layer of the olfactory bulb of both hemispheres. At the level of the subventricular zone, few MSCs were detected in both hemispheres. In the severely damaged ipsilateral hippocampus, many cells were present, whereas no MSCs were detected in the contralateral hippocampus.

Sensorimotor function and brain damage. Exposure to HI induced a preference for the use of the unimpaired forepaw in

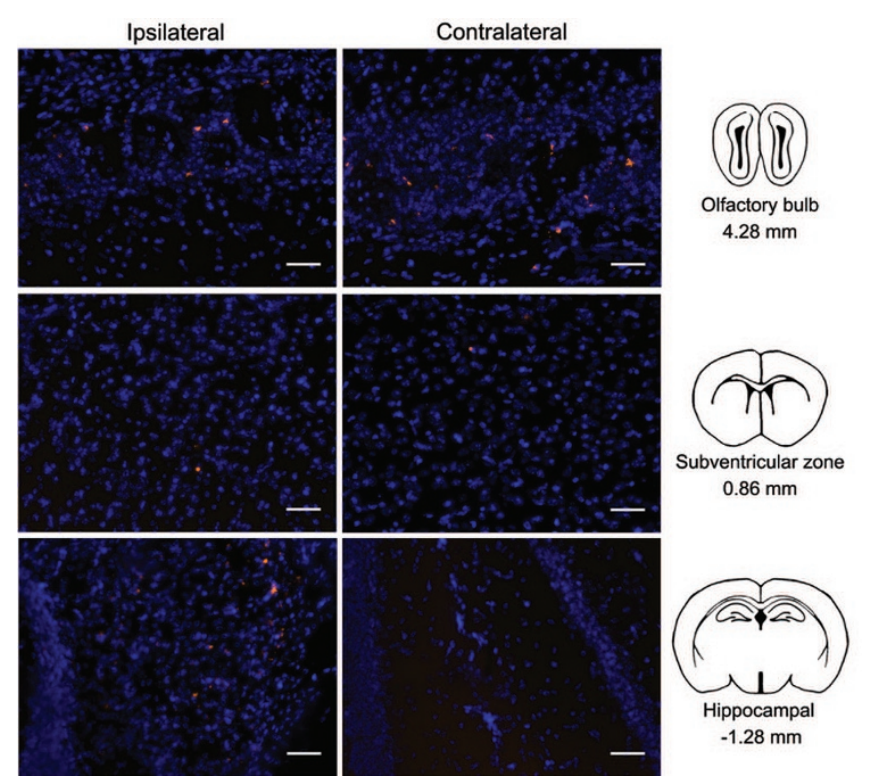

Figure 1. MSC location in brain after intranasal administration. Brain sections $18 \mathrm{~d}$ after intranasal administration of MSC. Sections were stained with DAPI (blue) to visualize all nuclei. MSCs (red) were found in both the ipsiand contralateral olfactory bulb. Few cells could be found in both hemispheres at the level of the subventricular zone. In the contralateral hippocampus, no MSC could be found. However, in the ipsilateral hippocampus, which is damaged, many MSCs are present. Scale bar represents $50 \mu \mathrm{m}$.

the cylinder rearing test when performed at 10, 21, and $28 \mathrm{~d}$ after HI. After intranasal application of MSC, forepaw preference was significantly reduced, indicating restoration of sensorimotor function (Fig. 2A). Intranasal administration of MSC at $10 \mathrm{~d}$ after $\mathrm{HI}$ also significantly reduced neuronal (34 $\pm 4 \%$ reduction of MAP2 loss; $p<0.05$ ) and white matter (37 $\pm 8 \%$ reduction of MBP loss; $p=0.05$ ) loss at $28 \mathrm{~d}$ after HI (Fig. $2 B$ and $C$ ). Vehicle treatment, i.e. hyaluronidase followed by saline, had no effect on sensorimotor function and lesion size.

Gene expression of MSC after culture with brain extracts. Transplantation of only $5 \times 10^{5}$ MSC resulted in a robust reduction in lesion volume. However, analysis of expression of NeuN, S100, or Iba-1 showed that cells of transplant origin had not differentiated into mature neurons, astrocytes, or microglia (data not shown). Therefore, we hypothesized that MSC may respond to signals in the ischemic brain by adapting 

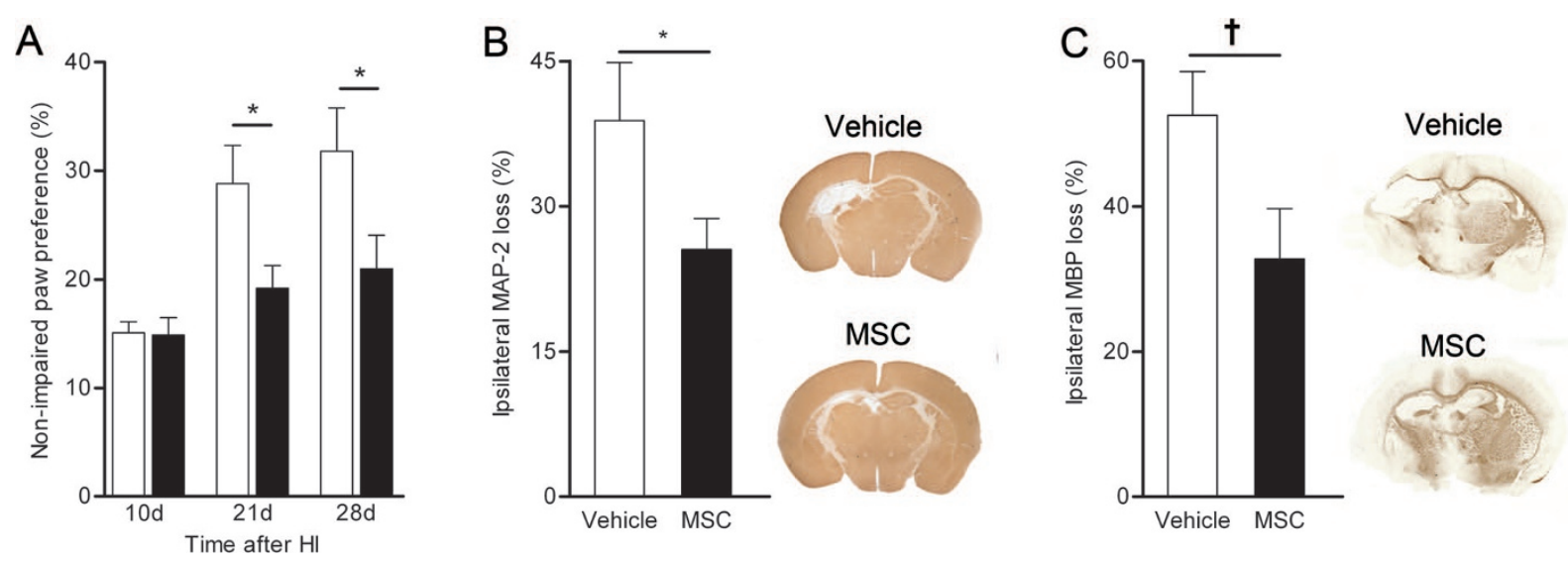

Figure 2. Sensorimotor function and brain damage after intranasal transplantation of MSC. Preference for use of the impaired paw was determined as a measure of sensorimotor function $(A)$. Quantification of MAP2 $+(B)$ and $\mathrm{MBP}+(C)$ area loss expressed as ratio ipsi- or contralateral area after intranasal administration of MSC at $10 \mathrm{~d}$ after HI. White bars: vehicle treated animals $(n=8)$; black bars: MSC-treated animals $(n=12)$. Data represent mean \pm SEM $\left({ }^{*} p<0.05\right.$; ${ }^{\dagger} p=$ $0.05)$.
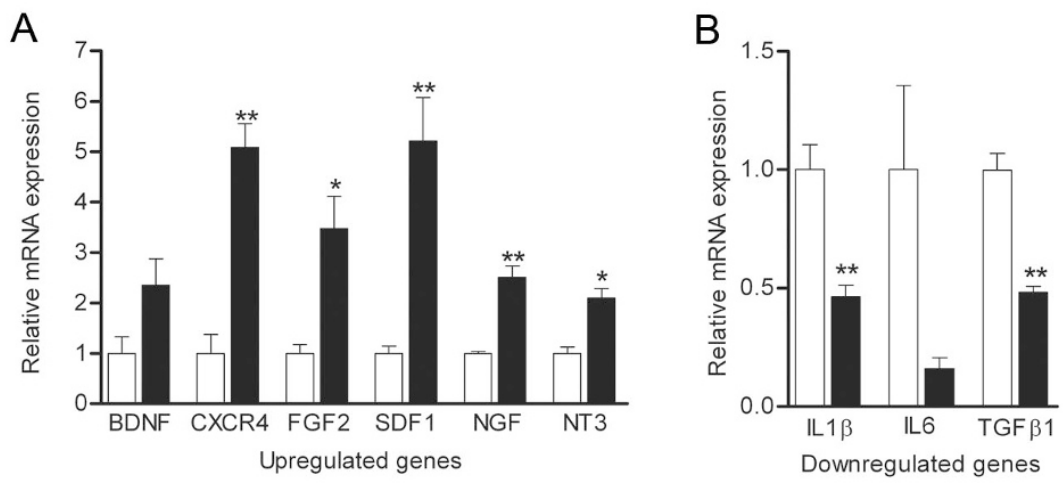

Figure 3. Changes in mRNA levels growth factors in MSC cultured with brain extracts. MSCs were cultured for $72 \mathrm{~h}$ with brain extracts obtained from sham-operated mice (white bars) or HI mice (black bars; $n=3$ per group). Culture of MSC with HI brain extracts induced increased mRNA expression of CXCR4, FGF2, SDF-1, NGF, and NT3 $(A)$ and down-regulation of IL1 $\beta$ and TGF $\beta 1(B)$ as determined by quantitative real-time RT-PCR. Data represent mean $\pm \operatorname{SEM}(* p<0.05, * * p<0.001)$. their expression of growth factors to stimulate endogenous repair processes. To test this hypothesis, MSCs were cultured in conditioned medium containing brain extracts from shamoperated animals or HI animals obtained at $10 \mathrm{~d}$ after HI. After culturing the MSC with the brain extracts for $72 \mathrm{~h}$ in vitro, mRNA expression of growth and differentiation factors was analyzed by qPCR. In response to HI brain extracts compared with sham brain extracts, MSC significantly up-regulated expression of chemokine (C-X-C motif) receptor 4 (CXCR4), fibroblast growth factor 2 , nerve growth factor, neurotrophin-3, and stromal cell-derived factor (SDF)-1, whereas expression of IL1 $\beta$ and transforming growth factor beta 1 (TGF $\beta 1$ ) by MSC was down-regulated (Fig. 3). mRNA expression of brain-derived neurotrophic factor and interleukin (IL)-6 was not changed by MSC cultured with HI brain extracts.

\section{DISCUSSION}

This study shows for the first time that nasally applied MSCs improve functional outcome and reduce gray and white matter loss in a model of neonatal HI brain damage. These findings indicate that the nasal route is an efficient route for stem cell transplantation after brain injury in the neonate.

Improved outcome after MSC treatment has been attributed to the inhibition of inflammatory processes that could contribute to damage, the differentiation of transplanted MSC into new neurons, and the stimulation of endogenous repair processes. In this study, MSC were applied via the nasal route at $10 \mathrm{~d}$ after HI. At this time point after HI, maximal reduction in MAP2 and MBP staining has already been obtained. Therefore, we anticipate that inhibition of injurious processes or prevention of damage are not the major mechanisms underlying the marked improvement in functional outcome and reduction in lesion size, but that MSC transplantation at this time point rather leads to regeneration of gray and white matter.

At d 28 after HI, which is $18 \mathrm{~d}$ after bilateral intranasal administration, MSCs were still present in the brain. However, it is hard to imagine that the relatively low number of transplanted MSC will exclusively be responsible for the profound reduction in lesion size that we observed. Moreover, no differentiation of transplanted MSC into a mature cerebral cell type was observed. This is in line with previous reports showing very limited differentiation of MSC into cerebral cell types after transplantation directly into the ischemic brain $(9,12,18)$. Therefore, we propose that the major mechanism underlying the improved outcome after nasal MSC treatment is enhancement of endogenous repair mechanisms. This notion is in line with results from our earlier studies in which we showed that in intracranial administration of MSC stimulated proliferation and/or survival of newly formed cells of host origin (12). Moreover, we showed that intracranial MSC 
transplantation stimulated differentiation of new cells into neurons and oligodendrocytes (12).

It is of particular interest that at the level of the hippocampus, MSCs were predominantly found in the ipsilateral and lesioned hemisphere after intranasal delivery. These findings indicate that on intranasal administration, MSCs migrate to the lesion site not only from the ipsi- but also from the contralateral hemisphere. This migration is probably effected by chemokines secreted at the site of injury such as SDF-1. SDF-1 is up-regulated after HI brain injury near the lesion site and is expressed for at least $14 \mathrm{~d}$ after induction of the injury (19). Furthermore, we show here that MSCs increase the expression of CXCR4 and the receptor for SDF-1, as a response to the ischemic environment in the brain.

Directed migration of nasally applied MSC to the lesion site may have the advantage that MSCs are allowed to adapt better to the milieu in the brain then, e.g. after intracranial administration leading to better MSC survival. This notion is supported by our finding that MSC applied intracranially, directly adjacent to the lesion site, could not be detected anymore in the brain at d 28 after HI, which is $18 \mathrm{~d}$ after administration (7).

As described above, we propose that the reduction of lesion size and improved functional outcome induced by intranasal or intracranial administration of MSC after HI are mainly mediated by stimulation of endogenous repair. We show here that MSCs express a number of growth and differentiation factors that could contribute to stimulation of repair. Moreover, MSC cultured with HI brain extracts obtained at $10 \mathrm{~d}$ after HI change their gene expression of several secreted factors that are known to regulate cell proliferation and differentiation and to improve outcome after brain injury (2024). Although it is not possible to define the exact contribution of each of these factors to repair processes, we can conclude that a trophic milieu, including brain-derived neurotrophic factor, fibroblast growth factor 2, neurotrophin, and nerve growth factor, is provided by MSC when in contact with signals provided by the damaged brain. It is also of interest that the proinflammatory cytokines such as IL- 1 and IL-6 are down-regulated by MSC after interaction with the ischemic brain, which may also contribute to controlling proper repair.

The setbacks that come with i.v. or intraarterial transplantation of MSC, such as potential loss of cells in the periphery and systemic immunosuppression, are avoided by nasal application. We demonstrate here that MSCs applied via the nasal route have therapeutic effects for repairing the neonatal brain after a hypoxic-ischemic insult. Therefore, we propose that this route of administration provides an effective alternative allowing frequent MSC treatments with minimal burden for the patient.

\section{REFERENCES}

1. Volpe JJ 2001 Neurology of the Newborn. W.B. Saunders, Philadelphia, PA

2. Ferriero DM 2004 Neonatal brain injury. N Engl J Med 351:1985-1995
3. Gluckman PD, Wyatt JS, Azzopardi D, Ballard R, Edwards AD, Ferriero DM, Polin RA, Robertson CM, Thoresen M, Whitelaw A, Gunn AJ 2005 Selective head cooling with mild systemic hypothermia after neonatal encephalopathy: multicentre randomised trial. Lancet 365:663-670

4. Shankaran S, Laptook A, Wright LL, Ehrenkranz RA, Donovan EF, Fanaroff AA, Stark AR, Tyson JE, Poole K, Carlo WA, Lemons JA, Oh W, Stoll BJ, Papile LA, Bauer CR, Stevenson DK, Korones SB, McDonald S 2002 Whole-body hypothermia for neonatal encephalopathy: animal observations as a basis for a randomized, controlled pilot study in term infants. Pediatrics 110:377-385

5. Chen J, Li Y, Wang L, Lu M, Zhang X, Chopp M 2001 Therapeutic benefit of intracerebral transplantation of bone marrow stromal cells after cerebral ischemia in rats. J Neurol Sci 189:49-57

6. Kurozumi K, Nakamura K, Tamiya T, Kawano Y, Ishii K, Kobune M, Hirai S, Uchida H, Sasaki K, Ito Y, Kato K, Honmou O, Houkin K, Date I, Hamada H 2005 Mesenchymal stem cells that produce neurotrophic factors reduce ischemic damage in the rat middle cerebral artery occlusion model. Mol Ther 11:96-104

7. van Velthoven CT, Kavelaars A, van Bel F, Heijnen CJ 2010 Mesenchymal stem cell treatment after neonatal hypoxic-ischemic brain injury improves behavioral outcome and induces neuronal and oligodendrocyte regeneration. Brain Behav Immun 24:387-393

8. Iihoshi S, Honmou O, Houkin K, Hashi K, Kocsis JD 2004 A therapeutic window for intravenous administration of autologous bone marrow after cerebral ischemia in adult rats. Brain Res 1007:1-9

9. Yasuhara T, Hara K, Maki M, Mays RW, Deans RJ, Hess DC, Carroll JE, Borlongan CV 2008 Intravenous grafts recapitulate the neurorestoration afforded by intracerebrally delivered multipotent adult progenitor cells in neonatal hypoxic-ischemic rats. J Cereb Blood Flow Metab 28:1804-1810

10. Lee JA, Kim BI, Jo CH, Choi CW, Kim EK, Kim HS, Yoon KS, Choi JH 2010 Mesenchymal stem-cell transplantation for hypoxic-ischemic brain injury in neonatal rat model. Pediatr Res 67:42-46

11. Yasuhara T, Matsukawa N, Yu G, Xu L, Mays RW, Kovach J, Deans RJ, Hess DC, Carroll JE, Borlongan CV 2006 Behavioral and histological characterization of intrahippocampal grafts of human bone marrow-derived multipotent progenitor cells in neonatal rats with hypoxic-ischemic injury. Cell Transplant 15:231-238

12. Li Y, Chen J, Chen XG, Wang L, Gautam SC, Xu YX, Katakowski M, Zhang LJ, Lu M, Janakiraman N, Chopp M 2002 Human marrow stromal cell therapy for stroke in rat: neurotrophins and functional recovery. Neurology 59:514-523

13. Qu R, Li Y, Gao Q, Shen L, Zhang J, Liu Z, Chen X, Chopp M 2007 Neurotrophic and growth factor gene expression profiling of mouse bone marrow stromal cells induced by ischemic brain extracts. Neuropathology 27:355-363

14. van Velthoven CT, Kavelaars A, van Bel F, Heijnen CJ 2009 Regeneration of the ischemic brain by engineered stem cells: fuelling endogenous repair processes. Brain Res Rev 61:1-13

15. Danielyan L, Schafer R, Ameln-Mayerhofer A, Buadze M, Geisler J, Klopfer T, Burkhardt U, Proksch B, Verleysdonk S, Ayturan M, Buniatian GH, Gleiter CH, Frey WH 2009 Intranasal delivery of cells to the brain. Eur J Cell Biol 88:315-324

16. Nijboer CH, Kavelaars A, Vroon A, Groenendaal F, van Bel F, Heijnen CJ 2008 Low endogenous G-protein-coupled receptor kinase 2 sensitizes the immature brain to hypoxia-ischemia-induced gray and white matter damage. J Neurosci 28:33243332

17. van der Kooij MA, Ohl F, Arndt SS, Kavelaars A, van Bel F, Heijnen CJ 2010 Mild neonatal hypoxia-ischemia induces long-term motor- and cognitive impairments in mice. Brain Behav Immun 24:850-856

18. Chen J, Li Y, Katakowski M, Chen X, Wang L, Lu D, Lu M, Gautam SC, Chopp M 2003 Intravenous bone marrow stromal cell therapy reduces apoptosis and promotes endogenous cell proliferation after stroke in female rat. J Neurosci Res 73:778-786

19. Rosenkranz K, Kumbruch S, Lebermann K, Marschner K, Jensen A, Dermietzel R, Meier C 2010 The chemokine SDF-1/CXCL12 contributes to the 'homing' of umbilical cord blood cells to a hypoxic-ischemic lesion in the rat brain. J Neurosci Res 88:1223-1233

20. Frielingsdorf H, Simpson DR, Thal LJ, Pizzo DP 2007 Nerve growth factor promotes survival of new neurons in the adult hippocampus. Neurobiol Dis 26:47-55

21. Kawamata T, Ren J, Cha JH, Finklestein SP 1999 Intracisternal antisense oligonucleotide to growth associated protein- 43 blocks the recovery-promoting effects of basic fibroblast growth factor after focal stroke. Exp Neurol 158:89-96

22. Sasaki M, Radtke C, Tan AM, Zhao P, Hamada H, Houkin K, Honmou O, Kocsis JD 2009 BDNF-hypersecreting human mesenchymal stem cells promote functional recovery, axonal sprouting, and protection of corticospinal neurons after spinal cord injury. J Neurosci 29:14932-14941

23. Schäbitz WR, Schwab S, Spranger M, Hacke W 1997 Intraventricular brain-derived neurotrophic factor reduces infarct size after focal cerebral ischemia in rats. J Cereb Blood Flow Metab 17:500-506

24. Zhang ZH, Wang RZ, Wang RZ, Li GL, Wei JJ, Li ZJ, Feng M, Kang J, Du WC, Ma WB, Li YN, Yang Y, Kong YG 2008 Transplantation of neural stem cells modified by human neurotrophin-3 promotes functional recovery after transient focal cerebral ischemia in rats. Neurosci Lett 444:227-230 